\title{
El poder en las relaciones de género desde la perspectiva de las mujeres ${ }^{1}$
}

Daniel Martínez Esquivel ${ }^{2}$

Institución: Universidad de Costa Rica

\section{CÓMO CITAR}

Martínez, D. (2014). El poder en las relaciones de género desde la perspectiva de las mujeres. Rev. Enfermería Actual de Costa Rica, 27, 1-19.

DOI: http://dx.doi.org/10.15517/revenf.v0i27.16267

\section{RESUMEN}

Introducción. El poder está determinado por las relaciones interpersonales, por tanto, hombres y mujeres tienen poder. Sin embargo, el poder en el sistema de las mujeres se ve disminuido por la cultura y los valores sociales, lo cual provoca desigualdad e inequidad que afecta directamente su salud mental, al punto de que disminuye su participación y empoderamiento social.

Método. La presente investigación buscó responder a la pregunta ¿cuál es la influencia del poder en las relaciones de género de un grupo de mujeres? Para lo cual, se planteó una investigación cualitativa fenomenológica, la muestra se tomó a conveniencia. En cuanto a los datos, se recolectaron mediante la técnica de grupo focal y su análisis se efectuó desde un enfoque interpretativo que incluyó una triangulación entre la teoría, la información obtenida y la posición del investigador.

Resultado. La principal función que ejerce el sistema de las mujeres es el trabajo doméstico, sin embargo, tienen oportunidades de educación y de trabajo. La toma de decisiones se limita al hogar y se da tan solo frente a personas que tienen un rol inferior. Las relaciones interpersonales son buenas siempre y cuando ejerzan un rol de mujer y madre socialmente aceptado.

Conclusión. El sistema de mujeres tiene poder pero está determinado por la cultura, de modo que las mujeres todavía sufren del poder de dominación de la sociedad patriarcal, por tanto, sus oportunidades de desarrollo disminuyen y sus funciones sociales se determinan a partir de su clase, posición en el sistema familiar, educación y producción económica.

Palabras clave: Enfermería; género; mujeres; poder; salud mental.

\footnotetext{
${ }^{1}$ Fecha de recepción: 13 de enero del $2014 \quad$ Fecha de aceptación: 10 de setiembre del 2014

2 Escuela de Enfermería. Universidad de Costa Rica. Costa Rica. Correo electrónico: dtinez@gmail.com
} 


\section{The power in gender relations from the perspective of women ${ }^{1}$}

Daniel Martínez Esquivel ${ }^{2}$

Institution: University of Costa Rica.

\section{CITED}

Martínez, D. (2014). The power in gender relations from the perspective of women. Rev. Enfermería Actual de Costa Rica, 27, 1-19. DOI: http://dx.doi.org/10.15517/revenf.v0i27.16267

Introduction. Power is determined by interpersonal relationships so, both, men and women, have power. However, the power in the system of women is diminished by the culture and values of our society causing inequality and inequity; it directly affects their mental health by decreasing their social participation and social empowerment.

Method. This research seeks to answer what is the influence of power in gender relations of a group of women? To answer this problem this research is a qualitative phenomenological investigation, a convenience sample was taken, the data was collected through focus group technique and analyzed from an interpretative approach performing a triangulation between theory, the information obtained and the position of the researcher.

Result. The primary function that the system of women is domestic work, however, has opportunities for education and work. Decision making is limited to home and faces people who have a lower role. Relationships are good provided that it exercises a role of wife and mother socially accepted.

Conclusion. The system has power but women is determined by culture, women still suffer the power of domination of patriarchal society so their development opportunities are diminished and social roles of women are determined by their class, their position in the family system, education and economic output.

Key words: mental-health; nursing; gender; power; women.

\footnotetext{
${ }^{1}$ Date of receipt: January 13, 2014

Date of acceptance: September 10, 2014

${ }^{2}$ School of Nursing. University of Costa Rica. Costa Rica. E-mail: dtinez@gmail.com
} 


\section{INTRODUCCIÓN}

Debido a que este es un estudio sobre el poder, hay que referirse a Foucault (1991), quien menciona el poder es incomprensible, si antes no se entiende al sujeto y su contexto, debido a que mientras el sujeto mantenga relaciones con otras personas y con la sociedad, se desarrollarán relaciones de poder. Si no existen relaciones, el poder tampoco existe. Estudiar el poder significa conocer el contexto social, medio en el que se desarrolla (el poder) a través de las relaciones entre el todo. Existe el poder en la política, la economía, el género, la educación, la familia y en muchos otros gobiernos ${ }^{1}$.

Para Foucault (1991), el poder es aquello que se ejerce sobre las cosas y tiene la habilidad para modificarlas pero, principalmente, su característica es que condiciona las relaciones entre los individuos, lo cual puede ser favorable o desfavorable, por ejemplo, existen los programas de seguridad social, mas también, la desigualdad y la inequidad. Igualmente, los poderes condicionan la salud de la gente, ya que su ejercicio puede estructurar y transformar a un individuo, una familia, una sociedad e, incluso, al mundo, mejorándolo o afectándolo. El ejercicio del poder permite comprender los contextos sociales y, a través de esta comprensión social, se puede entender la salud (Foucault, 1991). Dado lo anterior, para la Enfermería en Salud Mental es imprescindible conocer la situación actual de la sociedad en la que se desarrolla, puesto que facilitará la comprensión de las relaciones de poder existentes y las áreas de acción que se debe seguir para mejorar la calidad de vida de todos.

Como parte del entendimiento del sujeto y su contexto (el poder), y su relación con la salud, Wilkinson y Marmot (2003) mencionan que,

La atención médica puede prolongar la supervivencia después de algunas enfermedades graves, pero las condiciones sociales y económicas que determinan que las personas se enfermen o no son más importantes para las ganancias en salud de la población en general (p.6).

Lo anterior significa que la dinámica mundial está determinando la salud de las personas; es decir, no se puede pensar en salud sin antes dirigir la mirada a la política, la economía, la cultura y los valores de los diferentes contextos sociales; por ende, los profesionales en salud no pueden conformarse con atender la consecuencia y la enfermedad, pero ignorando todas sus causas, máxime que hoy la salud no es biológica, sino social, puesto que las causas más importantes que definen la salud de las personas están relacionadas con el ejercicio del poder.

Al respecto, para Neuman y Fawcett (2002), la salud es un proceso dinámico entre el individuo y el ambiente, donde el bienestar es el mayor nivel de salud posible y la enfermedad, el menor. El mayor nivel de salud se alcanza con la estabilidad de los sistemas que integran la vida, perspectiva desde la que tener salud significa acceder a todas las condiciones de bienestar que suponen desarrollo personal: alimentación variada y balanceada, educación de calidad, trabajo, transporte saludable, igualdad de género, entre otras, sin embargo, no siempre se cuenta con tales condiciones.

\footnotetext{
${ }^{1}$ Foucault se refiere a "gobierno" como la forma en que la conducta de los individuos o de los grupos puede ser dirigida, por ejemplo gobierno político, gobierno de la familia, gobierno de la salud. (Foucault, 1991, p. 86)
} 
La Organización Panamericana de la Salud (OPS, 2007), enfatiza que “...las desigualdades, la pobreza, la explotación, la violencia y la injusticia están entre las causas más importantes de los problemas de salud que afectan a la gente" (p.3), por consiguiente, en la comunidad es donde se encuentran los verdaderos problemas, determinantes sociales de la salud, que definen que las personas se enfermen o no, meollo al se deben dirigir todos los esfuerzos de atención en salud.

En cuanto a la salud mental, es

la base para el bienestar y el funcionamiento efectivo del individuo y de la comunidad (...) disminuida por la pobreza, la guerra y la desigualdad, las migraciones, el desplazamiento, la discriminación racial, la economía y los conflictos familiares e individuales, que son las principales causas del sufrimiento mental (Malvárez, 2009, p.168-170).

Tras establecer una relación entre ambos conceptos, se comprender la importancia de la atención en salud mental para el mejoramiento de las condiciones de vida de todos, máxime considerando que, según la OPS (2007), la salud es un derecho humano indispensable para que cada persona desarrolle su proyecto de vida.

Tras indicar lo anterior, es válido afirmar que,

la salud se construye dentro de un sistema de diferenciaciones, a partir de las relaciones interpersonales y su interacción con el medio; este sistema permite a un sujeto actuar sobre los demás, porque la ley se lo permite, por una cuestión cultural, por diferencias económicas, por la educación que tiene o por el simple hecho de ser hombre (Foucault, 1991, p.93).

Para poder ejercer su función de manera óptima, el profesional de enfermería no pueden ignorar dicha premisa, puesto que significaría que no es sensible a las necesidades de cuidado que el hombre y la mujer precisan en sus diferentes etapas de desarrollo. Por el contrario, la Enfermería debe posicionarse en la comunidad, comprender las relaciones de poder que se tejen a lo interno de ese sistema para impedir, cuidar y resolver los problemas a través de la promoción de la salud y la prevención de la enfermedad, de manera que contribuya al desarrollo de la sociedad (Malvárez, 2007).

De acuerdo con Neuman et al. (2002), la Enfermería es la única profesión de la salud capaz de atender todas las variables que afectan a las personas en su entorno. Su objetivo es valorar a la persona en relación con el ambiente para mantener el nivel más óptimo de salud del individuo, la familia y la comunidad a partir de la prevención; por ende, valora el todo como un sistema compuesto por subsistemas.

El trabajo en salud, en especial el trabajo de Enfermería, está ligado a comprender los contextos sociales para brindar una atención de calidad a partir del derecho al mayor nivel de salud posible, la equidad y la solidaridad. A partir de estos valores, la Enfermería en Salud Mental debe incorporar elementos como el primer contacto, la atención integrada y continua, el abordaje holístico de la familia, la orientación comunitaria, así como los cuidados apropiados, enfatizando la promoción y la prevención. 
Los conceptos anteriores justifican las acciones de la Enfermería en Salud Mental y permiten establecer una relación entre el estudio del poder en las familias y la salud, al punto de validar la perspectiva de promoción y prevención como estrategias que crean condiciones de bienestar para todos. Al respecto, Neuman et al. (2002) mencionan que para alcanzar el mayor nivel de salud posible, en cualquiera de los tres niveles de atención, los enfermeros deben enfocarse en la reducción de riesgos, la prevención, la protección y el mantenimiento del sistema individual o colectivo.

Dado que las relaciones de género son parte del sistema familiar, el tema se incluye en la agenda mundial, debido a que es una de las áreas fundamentales en las que se debe trabajar para mejorar la calidad de vida global. Para concretar dicho fin, la Enfermería en Salud Mental debe ejercer liderazgo en las comunidades y ser la abanderada de la salud; al respecto, Grace (2000) mencionada por Malvárez (2005), se refiere a la importancia de la Enfermería en la actualidad al afirmar que "sin Enfermería, será imposible lograr una atención adecuada en cantidad y calidad" (p.2); más si su currículo le permite ser sensible y comprender las necesidades de las personas y la comunidad para crear condiciones de bienestar mental en la sociedad.

La presente investigación se enfocó en el tema de las relaciones de poder a partir de un enfoque desde Enfermería en Salud Mental. Para analizar esta problemática se utilizó la Teoría de Sistemas de Neuman, la cual se basa en un proceso holístico enfocado en la percepción, orientación y motivación de la persona respecto del bienestar y su interacción dinámica con el ambiente (Neuman et al., 2002).

Neuman et al. (2002) propusieron una teoría que brindara la estructura, la organización y la dirección necesarias para que intervenir desde la Enfermería fuera lo suficientemente flexible para proporcionar atención adecuada a la persona. Según Neuman (1995), propone que la persona sea vista como "un cliente o sistema cliente" (p.15), sin embargo, de acuerdo a los principios de Bioética planteados en esta investigación, no se utilizará esa connotación, por lo que se hará referencia a la persona y no al cliente, considerando su complejidad. Este modelo permite valorar a la persona de acuerdo con todos los determinantes que definen su salud, con lo que se comprende el fenómeno que se desea a estudiar a partir de los significados que las participantes brindan.

La teoría propone que cada fenómeno es un sistema abierto que se mantiene en constante intercambio de energía e información con el ambiente y consigo mismo (Neuman et al, 2002, p.12), consigna desde la que consigna se identificó al sistema de las mujeres como sujeto de estudio.

De igual forma, se utilizó la perspectiva teórica de género, debido a que, como mencionan Gómez y Ortiz (2005), “...abordar la teoría de género permite comprender de un modo más amplio el vínculo de la socialización de género y las relaciones de poder con la construcción social de la salud” (p.52), de modo que sea posible estudiar el poder y el cómo se estructura las relaciones de género en un grupo de mujeres.

De acuerdo con lo expuesto, esta investigación pretendió responder al siguiente problema: ¿Cuál es la influencia del poder en las relaciones de género de un grupo de mujeres? 


\section{Revista Electrónica Enfermería Actual en costa Rica}

\section{MATERIALES Y MÉTODO}

La población de estudio fue las mujeres madres o encargadas que tuvieran a su hijo o hija matriculada en la institución. Para delimitarla, en colaboración con la dirección, se eligieron 4 grupos de materno y se extendió una invitación a cada una de ellas, en la que se señaló el propósito de la investigación. Ésta fue atendida por 3 mamás o encargadas quienes participaron en el estudio.

El estudio se realizó en la Escuela Maternal Montessoriana, institución pública de enseñanza preescolar de San José centro, seleccionada por su ubicación, ya que las familias de las y los estudiantes matriculados habitan distintos sectores del área metropolitana y viven condiciones sociales muy diferentes, lo que enriqueció el análisis.

La presente investigación se enmarcó dentro de un enfoque cualitativo que, según Hernández, Fernández y Baptista (2010), es aquel en el que “...se recolectan datos sin medición numérica para descubrir o afinar preguntas de investigación en el proceso de interpretación.” (p.7). Este enfoque se concentra en los significados que le dan las personas al universo, ya que busca profundizar en un tema específico para comprenderlo. El método cualitativo utilizado fue el fenomenológico. En la fenomenología “...el investigador no conoce el problema a estudiar sino que, por medio de interrogantes, está interesado en la vivencia y experiencia subjetiva de quien lo vive." (Muñoz y Lorenzini, 2008, p.102).

Para la recolección de datos se utilizó el grupo focal. El grupo focal consiste en una o varias reuniones de grupo, de 3 a 10 personas, con quienes se conversó en torno a una temática específica, dirigida por el investigador el cual debe organizar al grupo, fomentar la participación y manejar las emociones para conseguir los resultados esperados. Se espera que los grupos se desarrollen en un ambiente cotidiano e informal; los objetivos son generar una opinión de grupo y analizar la interacción interpersonal (Hernández et al., 2010), además con dicha técnica, se pretendió conocer la opinión de las participantes respecto del problema de investigación, planteado a partir de cuatro interrogantes:

1. ¿Cuáles son las funciones que desempeña dentro del sistema familiar?

2. ¿Cómo se toman las decisiones dentro del sistema familiar?

3. ¿Cómo se solucionan los problemas dentro del sistema familiar?

4. ¿Cómo describe las relaciones interpersonales entre los miembros del sistema familiar?

Además, de las interrogantes se derivaron categorías y subcategorías de análisis que se mencionan a continuación:

1. Funciones en el sistema familiar.

a. Trabajo doméstico

b. Trabajo remunerado

c. Estudio

d. Responsabilidad económica

2. Toma de decisiones en el sistema familiar.

a. Autoridad

b. Participación activa

3. Solución de problemas en el sistema familiar. 

a. Conductas aprendidas
b. Estrés
c. Violencia

4. Relaciones interpersonales en el sistema familiar.
a. Comunicación
b. Apoyo social

Para el desarrollo del grupo focal, se elaboró una agenda para la sesión y una guía de interrogantes que fue validada por tres expertas en la temática. La guía de temáticas fue abierta, con el fín de que cada participante tuviera la posibilidad de expresarse con libertad (Hernández et al, 2010). La sesión tardó alrededor de 2 horas.

A partir de la información recolectada, las categorías y subcategorías fueron analizadas desde el paradigma interpretativo, con el que se busca acceder a una comprensión racional del ser humano en todas sus dimensiones, permite explicar el conocimiento subjetivo a partir de los datos objetivos (Ulin, Robinson y Tolley, 2006).

También se utilizó la triangulación entre la teoría basada en el Modelo de Sistemas de Neuman (2002), los postulados sobre el sujeto y el poder de Foucault (1991), el enfoque de género de acuerdo con el planteamiento de Lagarde (1995); la información recolectada y la posición ontológica del investigador.

\section{Consideraciones éticas}

Para la presente investigación se adoptó las siguientes consideraciones éticas propuestas en la Declaración Universal sobre Bioética y Derechos Humanos (2005), tanto por parte del investigador, como de la población de estudio:

1. La dignidad humana y derechos humanos: en este rubro, se respetó la dignidad del investigador y de los participantes a partir de los derechos humanos y las libertades fundamentales, enfocados siempre en el bienestar de todo individuo. Los intereses de la ciencia y de la sociedad nunca se antepusieron al bienestar del individuo.

2. El bienestar y efectos nocivos: con este proyecto, se pretendió crear un beneficio para el grupo a partir de la intervención de Enfermería en Salud Mental, por lo que fue favorable que las participantes conocieran previamente los beneficios y riesgos a los que se exponían, individual y colectivamente, al colaborar en la investigación.

3. Autonomía y responsabilidad individual: se respetó las decisiones que tomaron las personas para participar en el estudio, así como para abandonarlo en el momento en que lo consideraran oportuno.

4. Consentimiento informado, fue absolutamente esencial: la persona implicada tuvo la capacidad legal de dar su consentimiento, y de afirmar que se encontraba en una condición que le permitía tomar decisiones sin la intervención de ningún factor externo y con suficiente conocimiento y comprensión del propósito del estudio para tomar una decisión consciente. El deber y la responsabilidad de determinar la calidad del consentimiento informado recayó en el investigador a cargo de este proyecto. 
5. Respeto a la vulnerabilidad humana y la integridad personal: el investigador procuró controlar las situaciones a las que se enfrentaron los participantes con el fin de proteger y respetar su integridad.

6. Privacidad y confidencialidad: toda la información que se obtuvo se manejó con la confidencialidad necesaria, además se mantuvo la privacidad de las personas que colaboraron en el estudio. Los resultados se utilizaron de acuerdo con lo expuesto en el consentimiento informado.

7. Igualdad, justicia y equidad: el estudio se desarrolló por parte de una persona calificada en el tema, la cual tuvo la capacidad y autoridad de responder a cualquier duda que se generara durante el proceso de la investigación o tomar cualquier decisión que permitiera su mejoramiento, de manera que se garantizara que las personas tuvieran condiciones favorables para realizar sus aportes en un ambiente de igualdad de oportunidades para todos.

8. Responsabilidad social y salud: el planteamiento del estudio tuvo como finalidad crear condiciones de bienestar para el goce máximo de la salud de las familias representadas por la persona que participó., objetivo logrado a partir de acciones de promoción de la salud mental. Luego, al finalizar la investigación, el investigador tiene la obligación de publicar los resultados tal como se dieron, de lo contrario, no deben ser divulgados a la población en general, lo cual permitirá compartir los beneficios con otras comunidades que los pueden aprovechar.

\section{RESULTADOS}

En este apartado se presentan los resultados obtenidos en el grupo focal con el fin de responder al problema descrito, a partir de las categorías y subcategorías de análisis planteadas en la investigación.

Por un lado, según el Modelo de Sistemas de Neuman, es importante caracterizar las variables que influyen en el estado de salud de cada mujer participante inmersa en un sistema familiar. Se proponen 5 variables: fisiológica, psicológica, sociocultural, de desarrollo y espiritual.

De acuerdo a la variable fisiológica, el sexo de la mujer no recibe atributos de autoridad porque le pertenece al hombre, aún en hogares en los que la mujer debe asumir ese rol, existe otro que lo asume, "siempre le recuerdo (a mi papá) que mi hijo no tiene la figura paterna que es la figura de autoridad, entonces yo le decía a él que si yo le digo algo a mi hijo tiene que hacerlo para que me respete como la autoridad, y que si él (mi papá) me seguía contradiciendo mi hijo nunca me iba a respetar" (Grupo focal, 2013). Como se puede observar, las mujeres tampoco se conciben como una figura autoritaria y cuando la deben ejercer lo hacen en condiciones de desventaja.

En cuanto a la variable psicológica, las mujeres asumen un rol protector en sus hogares, porque se les relaciona con la protección, el cuidado y el amor, características que deben presentar, de lo contrario no serían mujeres. Una participante mencionó lo siguiente: "madrugar, aplancharles la ropa, meterlos al baño, alistarles el desayuno, guardarles la merienda. Esto lo hago en una hora, trato de hacerlo lo más rápido posible" (Grupo focal, 2013). 
En la variable sociocultural se muestra la desventaja de ser mujer en una sociedad patriarcal: "él ( $m i$ hermano) quiso agarrar el rol de papá. No le gustaba que yo saliera, no le gustaba nada" (Grupo focal, 2013), respuesta de la que se colige que el dominio de unos sobre otras inicia desde la familia.

En referencia a la variable de desarrollo, el sistema de las mujeres debe enfrentarse a los poderes, deberes $\mathrm{y}$ derechos que les fueron otorgados. Se considera que pueden pensar en otras cosas siempre y cuando cumplan lo que se les ha asignado, como se ejemplifica a continuación: "Yo paso todo el día en el trabajo, entre semana estudio también, yo voy a la universidad (estudia administración de empresas), entonces, este, es agotador" (Grupo focal, 2013).

Por último, la variable espiritual está directamente relacionada a las demás variables ya que ésta se refiere a la filosofía o estilo de vida que llevan las mujeres participantes dentro del sistema familiar. Al referirse a las variables, se comprende cuál es esta filosofía de vida que lleva cada una.

Por otro lado, de acuerdo a las categorías y subcategorías de análisis derivadas de las interrogantes planteadas en el grupo focal, se obtuvieron los siguientes resultados.

\section{Categoría funciones en el sistema familiar.}

Se identificaron subcategorías como el trabajo doméstico. En relación a esto, una participante menciona "todo... lavar, aplanchar, cocinar... bueno, no lavo todos los días, pero cocinar y aplanchar si" (Grupo focal, 2013); ese es el sentir que refleja las funciones que desempeña en el sistema familiar. De acuerdo con lo mencionado, el sistema de las mujeres ha adquirido roles que debe cumplir para funcionar de manera "adecuada" dentro de la sociedad, al punto de que el espacio de las mujeres ha sido reducido al hogar, en donde debe cumplir su proyecto de vida, como lo manifestó una de las participantes: "Bueno, también los oficios de la casa, soy la que cocino, la que limpio, la que doblo la ropa, lavo, aplancho, o sea todo" (Grupo focal, 2013). Al sistema de las mujeres se le ha asignado tanto trabajo que se les reduce las oportunidades de desarrollarse en otras áreas de su vida.

Tal situación se da porque existen muchas personas que piensan que las únicas responsables de esas tareas son las mujeres, aunque en muchos casos el trabajo de la mujer también es compartido con su pareja masculina: "Con mi esposo nos turnamos, digamos yo cocino, entonces él acomoda la cocina y yo seco los platos o yo aplancho mientras él está acomodando la cocina" (Grupo focal, 2013). Tal testimonio refleja situaciones de equidad e igualdad entre las parejas que se están desarrollando en la actualidad y que significan un rompimiento de paradigmas. Incluso, la mujer puede verse beneficiada por el aporte de otros hombres diferentes a su pareja: "mi papá me ayuda mucho, digamos lo que es cocinar, él cocina entre semana" (Grupo focal, 2013).

Otra subcategoría presente es el trabajo remunerado. Dentro de este panorama, el sistema de las mujeres ha tenido que luchar para tener una mayor participación en el ámbito laboral, externo al hogar, sin descuidar sus obligaciones por ser mujer. En relación con el tema, una de las participantes mencionó: "si todo está tranquilo (en el trabajo), salgo 5:30 p.m. o 6:00 p.m., llego a mi casa a alistar la merienda del día siguiente, a aplanchar, 
digamos si tengo que lavar los uniformes de mi hijo, los lavo pero casi siempre los lleva sucísimos" (Grupo focal, 2013). Aparte, cabe mencionar que para satisfacer sus necesidades básicas, las participantes deben generar un ingreso económico sin descuidar su rol de mujer y mamá en la casa.

A pesar de esto, en la actualidad, las condiciones y el costo de vida han obligado a la sociedad a abrir espacios en los que las mujeres puedan ser productivas, inclusive, en ocasiones, el sistema de las mujeres cuenta con el apoyo social de los miembros de la familia, tal como menciona una de las participantes "los dos trabajamos, entonces nos repartimos las cosas, prácticamente vamos mita y mita" (Grupo focal, 2013), caso en el que tanto el hombre como la mujer tienen una participación económica activa e igualitaria, sin importar que no cuenten las mismas oportunidades.

Relacionada al trabajo remunerado, se identificó la responsabilidad económica como otra subcategoría de análisis dentro de las funciones en el sistema familiar. En los casos en los que la familia es "unimaternal", la mujer debe asumir un doble rol social, el del hombre y el de la mujer, como ejemplifica una de las participantes: "lo que son gastos de mi hijo, sí son solo míos" (Grupo focal, 2013).

\section{Categoría toma de decisiones en el sistema familiar.}

Se derivaron 2 subcategorías: la autoridad y la participación activa las cuáles son interdependientes.

En los datos recolectados, se identificó que existe un ejercicio de autoridad y de la participación activa de la mujer-madre en la familia, una de las participantes mencionó: "digamos, yo cuando es una decisión de la casa, la que la toma soy yo" (Grupo focal, 2013), respuesta de la que se colige que, dentro del hogar, las mujeres tienen poderes que pueden ejercer de acuerdo con sus intereses.

Sin embargo, se debe tomar en cuenta la situación en que estas mujeres ejercen el poder ya que dos de las tres participantes forman parte de una familia "unimaternal". Se podría decir que el poder que ejercen se debe a que son el modelo de autoridad en el hogar, de modo que son las únicas capaces de dirigir a la familia, como lo afirma una de las participantes: "las decisiones las tomo yo, nada más..." (Grupo focal, 2013). Por ejemplo, una de las participantes comentó: "nosotros somos los dos. Nosotros todo nos lo consultamos, nos decimos si nos parece, si es bueno o no" (Grupo focal, 2013), contexto del que se desprende una relación de igualdad de oportunidades entre hombre y mujer.

\section{Categoría de solución de problemas en el sistema familiar.}

Las mujeres participantes ejercen su rol de poder reflejado en la autoridad, por lo que la resolución de problemas recae sobre ellas sin importar la presencia de hombres en la familia, como se refleja en la dinámica familiar de esta participante: "mi mamá siempre fue la autoridad, porque mi mamá es bravisima" (Grupo focal, 2013).

Además, de acuerdo con lo que mencionó la participante parece que las conductas aprendidas es una subcategoría presente: "Cuando peleo, bueno, mi esposo es muy callado, él no es de pelear, entonces me da más 
cólera porque él se queda callado" (Grupo focal, 2013). Al igual que su mamá, esta mujer es la autoridad en su casa frente a su esposo y a su hijo con quienes repiten la experiencia vivida con su papá y su mamá.

Lo anterior ha provocado inestabilidad en el sistema de las mujeres, perjuicio que se refleja en una disminución de su bienestar y salud mental: "soy muy estresada, yo todo lo tomo como más apecho de lo normal" (Grupo focal, 2013),

Esta construcción social podría traer consecuencias como el estrés en el momento de solucionar un problema en la familia: "yo sé, yo lo que tengo es eso, exploto muy rápido entonces intento controlarme" (Grupo focal, 2013). Esto ejemplifica al estrés como otra subcategoría presente en la información obtenida. Un mal manejo del estrés provocaría situaciones de violencia en la dinámica familiar, tal como lo menciona una de las participantes: "yo soy muy gritona y a veces lo que me dan ganas es de darle una nalgada, porque desde que nos levantamos es un pleito porque no se mete al baño o porque desayuna muy despacio" (Grupo focal, 2013).

No tener control sobre el estrés podría generar violencia dentro del sistema familiar, es por esta razón que la violencia es otra subcategoría de análisis presente en la solución de problemas en el sistema familiar. Sin embargo, cabe destacar que el objetivo no es calificar una situación cotidiana como un acto de violencia, sino demostrar que un mal manejo del estrés podría convertir situaciones, aparentemente normales, en otras que afecten todo el sistema familiar, su dinámica y desarrollo, hasta mostrar conductas que se salen de control: “ya si un día la chiquitilla hizo algo que no tenía que hacer y yo la agarro y le doy una nalgada, me pongo a llorar porque yo no puedo" (Grupo focal, 2013).

En cuanto al punto anterior, otra participante mencionó "yo pegarle a mi hijo, no puedo pegarle. Llegar a golpearla, por más que me diga (la gente), no puedo" (Grupo focal, 2013), acción que se opone a situaciones comunes que muchas mujeres han experimentado.

Otro aspecto importante es si las mujeres solucionan de la misma forma los problemas con sus hijos que con sus hijas, una de las participantes mencionó: "con mi hija no exploto (cuando hace algo que no debe hacer), con el que exploto es con mi hijo porque pelea mucho con la chiquitita" (Grupo focal, 2013). En apariencia la respuesta es no, sin embargo, podría tener una respuesta sociocultural.

\section{Categoría relaciones interpersonales en el sistema familiar.}

En la información recolectada se identificaron dos subcategorías: la comunicación y el apoyo social.

Debido a que el ser humano es estrictamente social siempre usa la comunicación, desde que nace hasta que muere; por consiguiente, en el momento en que la comunicación cambia, los beneficios del apoyo social empiezan a disminuir y la familia se vuelve disfuncional. Al respecto, una de las participantes menciona "con ellos (familia ampliada) es muy poco lo que tratamos porque cada uno tiene su aposento, yo vivo en el aposento de arriba" (Grupo focal, 2013), aquí se evidencia que en el momento en que cada miembro del sistema familiar guarda su espacio, la comunicación es compleja con los demás, lo cual dificulta la relación. 
Sin embargo, una participante afirma "mi esposo, nos llevamos muy bien porque somos como amigos, porque nos vacilamos, nos molestamos, vacilamos, cuando tenemos que pelear, peleamos" (Grupo focal, 2013), en este ejemplo se logra apreciar que, sin importar las circunstancias de la vida diaria, la familia puede mantener su funcionalidad independientemente de las relaciones de género que existan.

La funcionalidad de la familia de las participantes se refleja en la red social de comunicación que tiene significado para cada miembro, por ejemplo un padre afirmó: "con el embarazo le tengo más confianza, no sé, como que nos hicimos más amigos" (Grupo focal, 2013); en ese caso se puede observar una relación positiva padre e hija dentro del sistema familiar que refleja el efecto del apoyo social y la buena comunicación, que trae beneficios para su salud mental.

\section{DISCUSIÓN}

Desde el saber de la Enfermería, Neuman et al. (2002) propusieron una teoría que brinda la estructura, la organización y la guía necesarias para que la intervención de Enfermería en Salud Mental sea lo suficientemente flexible al atender de manera adecuada a la persona, considerando su complejidad. Este modelo permite valorar a la persona de acuerdo con todos los determinantes que definen su salud, de modo que facilita comprender el fenómeno que se desea estudiar a partir de los significados que las participantes brindaron. Esta teoría propone que cada fenómeno es un sistema abierto que se mantiene en constante intercambio de energía e información con el ambiente y consigo mismo. Según la propuesta de esta investigación, se identificó como sujeto de estudio el sistema de las mujeres.

Antes de analizar el sistema de las mujeres, se debe comprender el de género y su relación con el poder. En relación con Foucault (1991), el poder por sí no existe, sino que depende de otros factores como el sistema de género, el cual se define por las relaciones que establecen hombres y mujeres, en las que media un poder nocivo entre los sexos, que también puede utilizarse de manera positiva. Según Lagarde (1995), el problema del poder en el sistema de género radica en su disparidad y contextualización: es definido por el lugar que hombres y mujeres ocupan en el mundo.

Sin embargo, se debe aclarar que este poder no nace en la naturaleza sino que está moldeado por el contexto histórico en el que se desarrolla, por ello es que el poder en el mundo parece asimétrico, desigual e inequitativo, debido a la organización social patriarcal y poder que esta ejerce: se trata de una organización social determinada por la dominación masculina que se basa en la opresión sobre las mujeres (Lagarde, 1995).

La dominación se puede definir como injusta, puesto que aleja al sistema de las mujeres de su estabilidad de bienestar; es decir, las aleja de la salud, cuyas consecuencias son la infelicidad, el sufrimiento, los conflictos y las limitaciones del desarrollo personal (Lagarde, 1995).

El poder que cada uno o una posea depende de la persona, su condición de género, su familia, su educación, su proyecto de vida, su religión, en fin, por múltiples determinantes que definen quién es como individuo, aunque, lamentablemente, los deberes y derechos de los hombres y las mujeres están definidos por el género y el poder social dominante (Lagarde, 1995). 
ISST $1409-4568$

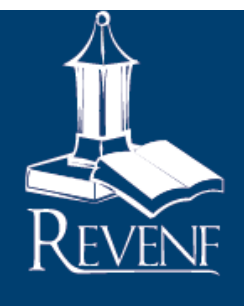

\section{Revista Electrónica Enfermería Actual en costa Rica}

Respecto del poder, otro aspecto importante que menciona Foucault (1991), es que no se puede comprender sin antes ver el contexto, es así como el hoy influye en el sistema de género al definir sus roles y poderes de acuerdo al sexo. En relación con lo anterior, Lagarde (1995) afirma que el contexto indica las actividades del sujeto, sus quehaceres y sus prohibiciones, las normas que lo determinan y los poderes que posee. En este contexto, el sistema de las mujeres se ve sometido al poder de dominio, el cual controla sus vidas a partir de juicios, castigos y perdón; el poder genera dependencia porque implica la necesidad de quien domina la relación porque tiene la verdad, la razón y la fuerza.

De acuerdo con el Modelo de Sistemas de Neuman, los sistemas en general y en este caso en particular -el sistema de las mujeres- están compuestos por cinco variables interactivas (Neuman et al., 2002), que engloban los múltiples determinantes que definen a las mujeres y su identidad, el bienestar general del sistema y permiten comprender el fenómeno de estudio, lo cual facilita la comprensión de su desarrollo sociohistórico y justifica la información obtenida a partir de las interrogantes planteadas en esta investigación.

Respecto de la variable fisiológica que, en cuanto al sistema de las mujeres, está definida por el sexo. Acerca del tema, Lagarde (1995), menciona que las marcas corporales reconocen dos tipos de cuerpo, hombre y mujer: al cuerpo de la mujer se le asignan roles y atributos que se debe desempeñar en el sistema de género, en la sociedad; pueden ser eróticos, económicos, sociales, culturales, psicológicos y políticos y definen lo que significa ser mujer en la actualidad. Se considera que ser mujer es sinónimo de desventaja y desigualdad y menos oportunidades de desarrollo; por ende, es más difícil alcanzar el éxito.

En cuanto a la variable psicológica, las mujeres están construidas para vivir por otros y quien no rechace esa premisa será excluida, desvalorizada, dañada, dado que solo es al ser poseída, dependiente y dominada, porque no se pertenece sino que pertenece a otros (Lagarde, 1995). Lo anterior les impide alcanzar su desarrollo personal ya que primero piensa en los demás y después en los demás, actitud que se refleja en los resultados, cuando una de las participantes mencionó todo lo que debe realizar antes de pensar en sí misma.

La variable sociocultural muestra que la sociedad está definida por la cultura patriarcal, afirmación que significa que las mujeres son oprimidas por los hombres y acerca de la que Lagarde (1995), comenta que la mitad de la humanidad, las mujeres viven en cautiverio sometidas a la opresión de género, mientras que la otra mitad, los hombres, tiene la posibilidad de ejercer formas de dominio, por lo menos sobre las mujeres. Tal desventaja ha provocado un aumento en la brecha de oportunidades para las mujeres, lo cual dificulta el acceso a la educación, al trabajo, incluso, a la socialización.

En la variable de desarrollo, sus oportunidades de alcanzarlo se enfrentan a la feminización de la pobreza, la discriminación, la subordinación y la violencia de hombres e instituciones (Lagarde, 1995), de modo que enfrentarse a su propio desarrollo personal implica el ejercicio de múltiples roles que primero la deben hacer mujer y luego todo lo demás que quiera ser.

Por otro lado, la variable espiritual, forma parte de la valoración holística que se realiza en cada sistema. Neuman et al. (2002), comenta que se refiere a las creencias e influencias que afectan a un sistema, las cuales ingresan al sistema, en el ciclo de entrada, en forma de energía hasta influir en la estabilidad de la estructura básica. Se considera que esta variable es fundamental para la valoración de cada sistema y está directamente relacionada al efecto de cada una de las otras en el sistema. 
Las variables se desarrollan en el ambiente interno y externo del sistema de las mujeres y permiten comprender el problema de estudio, planteado a partir de las interrogantes formuladas, las cuales se conciben como estresores internos y externos y están directamente relacionadas con el poder en las relaciones de género dentro de la familia y representan el ciclo de entrada.

Cada sistema posee mecanismos de amortiguación frente a las variables o determinantes de la salud, más el éxito de su funcionamiento definirá el bienestar y estabilidad que posee. Neuman et al. (2002), proponen que cada sistema tiene líneas de defensa y de resistencia que ejercen un mecanismo de protección con el fin de preservar su integridad. Sin embargo, se considera que estas variables han logrado traspasar las líneas de defensa, y peor aún, las líneas de resistencia al punto de desestabilizar la estructura básica del sistema de las mujeres y acercarlas a condiciones de desigualdad e inequidad que determinan su salud mental.

Por lo tanto, se considera que las variables que influyen en el sistema de las mujeres han traspasado todos sus mecanismos de defensa, lo cual afecta directamente su estructura básica. De acuerdo con Neuman et al. (2002), la estructura básica consiste en una serie de factores de supervivencia comunes en los sistemas, como por ejemplo sus características genéticas, sus fortalezas o sus debilidades. La influencia de estas variables ha favorecido el entorno creado en el que se desarrolla la vida cotidiana, el cual se refiere a una expresión simbólica del todo que refleja la movilización inconsciente de todas las variables de la persona, incluyendo los factores de la estructura básica, pensados para la integración, estabilidad e integridad de la persona (Neuman et al, 2002). El entorno creado es en donde las personas tienen necesidades de salud y en donde la Enfermería en Salud Mental debe enfocarse para brindar atención; en este caso, a partir de él, se logró comprender la información obtenida durante el grupo focal.

En referencia a las funciones en el sistema familiar, en su mayoría, los roles que desempeñan estas mujeres están determinados por su sexo, lo cual coincide con lo expuesto por Lagarde (1995), quien menciona que ser hombre o ser mujer está socialmente estructurado de acuerdo con los roles que se les asignan según lo que producen, intercambian y las relaciones que establecen, sin embargo, se puede observar algunas actividades que desempeñan estas mujeres y que les permiten tener mayor participación social.

Estas funciones se refieren al oficio o la limpieza, incluso, la persona que las desarrolla se le denomina la empleada o la porta, nombres dados porque esas actividades no son considerados un trabajo. Con tales palabras, según Vázquez y Murguialday (2000), se pretende ocultar que estas actividades son un trabajo importante, que ocasionaría problemas a la sociedad, en general, si no se hiciera. Este tipo de actividad es conocida como trabajo doméstico.

El trabajo doméstico puede definirse como aquella actividad humana que permite la producción de bienes y la prestación de servicios que aseguren el bienestar de los demás, el control del hogar y la reproducción de la sociedad (Vázquez et al., 2000). Este concepto permite identificar la relevancia de dicha actividad para la sociedad, debido a que muchas personas dependen de él para garantizar su crecimiento y desarrollo personal, educacional y laboral.

A pesar de que hay excepciones, Vázquez et al (2000), indican que, según los hombres, no les corresponden las tareas del hogar y que las mujeres tan solo deberían aspirar a que ellos ayuden con alguna 
"cosita"; dicho pensar es resultado de las relaciones intrafamiliares y no como un acto de caridad por parte de los hombres a las mujeres.

Siguiendo en el contexto de trabajo, se considera que el sistema de las mujeres en este ámbito es productivo, el problema surge en cuanto esa productividad no es importante para la sociedad patriarcal, al respecto, Lagarde (1995) menciona que las mujeres producen riqueza económica y social cuando logran preservar el medio, la casa o la familia, mas fuera de estas actividades, no existen espacios donde pueda participar, puesto que por su sexo no les corresponden.

En cuanto a la función de los hombres, Vázquez et al. (2000), mencionan que el lugar donde se elaboran las mercancías ha sido un espacio dominado por los hombres, por lo que se ha relegado el título de "ama de casa" única y exclusivamente a las mujeres. En un escenario como el anterior, Lagarde (1995) menciona que la mujer se enfrenta a la sociedad minimizada y sometida a la opresión patriarcal; no obstante, se prefiere considerar que la mujer que sale de su casa a trabajar es un ejemplo de resiliencia y emancipación ante una sociedad que le ha dado la espalda, y que otras mujeres deben tomar como ejemplo para superarse.

Finalmente, se podría decir que dicha dinámica está condicionada por las pocas oportunidades del sistema de las mujeres de usar sus habilidades y tomar sus propias decisiones, lo que, en definitiva, afectaría su bienestar y salud mental, al punto de generarle síntomas como el estrés y el estrés laboral (trabajo doméstico-trabajo). El estrés laboral contribuye a agravar las diferencias en salud entre las personas, mientras que la ausencia de enfermedad o la muerte prematura se relacionan con la posición que ocupan en la sociedad (Wilkinson et al., 2003). El sistema de las mujeres tiene menos oportunidades de trabajo remunerado de calidad que el sistema de los hombres, lo cual redunda en inequidades que disminuyen su salud.

Con respecto a la toma de decisiones en el sistema familiar, según Lagarde (1995), las mujeres que ejercen autoridad sobre los demás, lo logran con personas inferiores o que le son próximos, por ejemplo el esposo o un hijo. Dicha situación llevaría a pensar que bajo ninguna circunstancia, las mujeres podrán desarrollarse de manera plena, a pesar de ello no se pueden descartar casos en los que exista una relación de equidad entre ambos sexos.

También se podría pensar en una participación activa y plena de las mujeres en el hogar, donde asumen roles distintos a los que la cultura les ha asignado, cambio que, sin lugar a dudas, beneficiaría no solo a las mujeres, sino a sus familias, la comunidad y la sociedad en general (OMS, 2009). La participación de las mujeres representa una forma de emanciparse y liberarse de la opresión de género, con el fin de favorecer el desarrollo de su estructura básica, cuya consecuencia es la estabilidad general de su salud.

En la categoría solución de problemas en el sistema familiar, se evidencia la presencia de una lucha entre las personas por asumir el poder. Estas relaciones de poder intergenéricas están definidas por condiciones históricas de género, edad, saber, experiencia, entre otras (Lagarde, 1995); en este sentido, el poder de las mujeres participantes existe debido al rol que ejercen en la familia, como por ejemplo el cuidado de los hijos, el mantenimiento de la casa o la provisión económica que se relacionan a conductas aprendidas. 
Lagarde (1995) insiste que a pesar de estas funciones sociales, que en apariencia le brindan prestigio a la mujer, debido a su situación cultural y política siguen siendo presas del cautiverio al que las someten los hombres, el cual dificulta su desarrollo personal, reduce las oportunidades que tiene para estudiar, trabajar o tener salud afectando su estructura básica.

Esta situación genera aumento del estrés dentro del sistema provocando diferentes complicaciones y consecuencias, a pesar de que, de acuerdo con Lagarde (1995), culturalmente, las mujeres están ideologizadas para ser amorosas, dulces, débiles, de manera que los problemas podrían afectar su estructura básica de una forma más pronunciada. Se debe aclarar que esa respuesta socialmente aceptada, aunque no es algo natural, sino que son conductas culturalmente aprendidas. En cuanto al desarrollo del estrés, Wilkinson et al. (2003), mencionan que se debe a momentos en que la persona no puede tomar sus propias decisiones o ve disminuidas sus redes de apoyo, que también afecta a aquellas personas en situación inferior respecto de la jerarquía social.

En relación con la violencia, la OMS (2009), la define como,

el uso intencional de la fuerza o del poder físico, de hecho o como amenaza, contra uno mismo, otra persona o un grupo o comunidad, que cause o tenga muchas probabilidades de causar lesiones, muerte, daños psicológicos, trastornos del desarrollo o privaciones (p.5).

El concepto anterior permite ampliar la dimensión de violencia, en cuanto se refiera a otras áreas de la persona que puedan verse afectadas, más allá de un daño físico.

Se podría decir que a pesar de que la mayoría de mujeres ha sufrido dominio, hostigamiento, violencia o violación (Lagarde, 1995), esta conducta generalmente atípica en el sistema de las mujeres se debe a que las líneas de resistencia protegen a la estructura básica de adoptar comportamientos violentos.

En relación con el cuerpo, según Lagarde (1995), el de los hombres es poderoso y condenado a ser lo que no es la mujer, noción cultural conocida por el sistema de las mujeres ante la cual, en relaciones en las que debe ejercerse poder sobre los hombres, se toma una actitud de violencia para pasar por una fuente de autoridad. Sin embargo, esa conducta debe ser aprendida por la mujer, entonces, se estaría desarrollando como alguien que debe ejercer dominio sobre los demás, repitiendo el ciclo vicioso de la construcción de género.

Además, la estructura política especializa al ser humano por sexo, lo hace experto en actividades y funciones particulares para garantizar la continuidad del mundo (Lagarde, 1995), por lo que en la actualidad, ser hombre o ser mujer está definido por lo que la sociedad dicta como correcto, por los roles y los poderes que les son asignados.

La última categoría de análisis hace referencia a las relaciones interpersonales en el sistema familiar las cuales están mediadas por la dinámica que se tenga en la familia. Una dinámica familiar positiva tendrá un mejor impacto en el bienestar y salud mental de las personas que la integran, puesto que las buenas relaciones en la familia podrían significar un apoyo social básico para favorecer el desarrollo personal, acerca de lo que Wilkinson et al (2003), mencionan que "el apoyo social ayuda a darles a las personas los recursos emocionales y prácticos que necesitan" (p.21). 
El apoyo social se establece a partir de la comunicación, la cual se puede definir como el "proceso interpersonal en el que los participantes expresan algo de sí, a través de signos verbales o no verbales, con la intención de influir de algún modo en la conducta del otro u otra" (Madrid, 2005, p.98). En términos positivos, con este concepto se identifica que la comunicación no es estrictamente verbal, además es a través de ella que se puede modificar la conducta de otra persona.

Sin embargo, Stuart et al. (2006), plantean que el sistema familiar tiene la capacidad de ser funcional cuando logra tolerar las conflictos y adaptarse a cualquier circunstancia sin sufrir disfunción o desintegración de la familia, para lo que se deben desarrollar redes familiares fuertes. Las redes sociales de comunicación y obligaciones mutuas hacen que las personas se sientan amadas, apreciadas y cuidadas, hasta generar un efecto protector en su salud. (Wilkinson et al., 2003)

Para finalizar, la información obtenida, a partir de las interrogantes de investigación, permitió un acercamiento al problema planteado, ¿cuál es la influencia del poder en las relaciones de género de un grupo de mujeres? Ante esta problemática, se puede afirmar que el poder siempre influirá en las relaciones interpersonales de género en el sistema de las mujeres, porque el poder nace a partir de las relaciones entre las personas.

Sin embargo, se comprendió que el poder ejercido entre hombres y mujeres está determinado por la cultura de cada sociedad, independientemente de si el resultado es positivo o negativo. En nuestra sociedad, la influencia de poder genera desigualdades e inequidades que afectan principalmente a las mujeres, por lo que se les dificulta tener más oportunidades de participación social respecto de los hombres, diferencia que afecta su bienestar y salud mental.

Para la Enfermería en Salud Mental, el poder y las relaciones de género representan un problema de salud pública al que deben dirigirse acciones de promoción y prevención de la salud, con el fin de eliminar las desigualdades e inequidades del sistema de las mujeres. En relación con tal afirmación, Neuman et al. (2002), mencionan que las acciones de Enfermería deben retener, alcanzar y mantener la salud de las personas a partir de la prevención, para favorecer la estabilidad del sistema, por ende, lograr la estabilidad del sistema de las mujeres favorecerá su derecho a la salud mental por medio de la justica y participación social.

\section{CONCLUSIONES}

El poder existe a partir de las relaciones interpersonales, entonces, tanto hombres como mujeres tienen poder, aunque desigual, porque es atribuido por el sistema de género que depende de la cultura de cada sociedad.

Todas las mujeres sufren por el poder de dominación de la sociedad patriarcal independientemente de la comunidad a la que pertenezcan.

Las características asignadas al sistema de las mujeres, específicamente de las participantes, están determinadas por su clase, su posición en el sistema familiar, su educación y su producción económica.

Las características de las participantes les permiten tener más oportunidades y una mayor participación social que otras mujeres, lo cual favorece su bienestar y salud mental. 


\section{Revista Electrónica Enfermería Actual en costa Rica}

El principal rol que ejercen las participantes en el sistema familiar es el trabajo doméstico, pero también el trabajo remunerado y el estudio.

Los roles que ejercen las participantes promueven la adquisición de diferentes poderes en el sistema familiar, sobre hombres o mujeres, sin embargo, son influenciadas por el poder de dominación imperante.

Las participantes asumen la toma de decisiones en el sistema familiar, debido a las oportunidades que les brindan su posición en la familia, el tipo de familia o las conductas aprendidas de una generación a otra.

Las participantes forman parte de la solución de problemas en el sistema familiar, lo cual está determinado por el tipo de familia, la dinámica familiar o por conductas aprendidas.

El mal manejo del estrés en el sistema de las mujeres podría tornar en situaciones de violencia que afecten la dinámica familiar.

Las buenas relaciones interpersonales en el sistema familiar facilitan el ejercicio de poderes como autoridad, toma de decisiones o resolución de problemas.

\section{REFERENCIAS BIBLIOGRÁFICAS}

Foucault, M. (1991). El sujeto y el poder. Bogotá: Carpe Diem Ediciones.

Gómez, R., Ortiz, J. (2005). Salud de las mujeres, implicaciones de Enfermería. Colección de textos de Enfermería, salud integral de la mujer. San Salvador: JICA.

Hernández, R., Fernández, C., Baptista, P. (2010). Metodología de investigación. Quinta edición. México D.F.: McGraw-Hill Educación.

Lagarde, M. (1995). Género y poderes. San José: IEM-UNA.

Madrid, J. (2005). Los procesos de la relación de ayuda. Segunda Edición. Madrid: Editorial Desclée de Brouwer.

Malvárez, S. (2009). Promoción de la salud mental. Por Rodríguez, J. (Ed.). Salud Mental en la Comunidad. Segunda Edición. (133-134). Washington D.C.: OPS.

Malvárez, S. (julho, 2007). El reto de cuidar en un mundo globalizado. Texto y Contexto Enfermagem, año/vol. 16, número 003.

Malvárez, S. (septiembre, 2005). Recursos humanos en Enfermería: Desafíos para la próxima década. Revista Enfermería Universitaria, 2 (3).

Ministerio de Salud/OPS. (2004). Situación actual de la salud mental en Costa Rica. San José: OPS. 
Muñoz, L., Lorenzini, A. La fenomenología en la producción de conocimientos en Enfermería. En do Prado, M., de Souza, M., Carraro, T. (2008). Investigación cualitativa en Enfermería: contexto y bases conceptuales. Serie PALTEX Salud y Sociedad 2000 No.9. Washington D.C.: OPS.

Neuman, B., Fawcett, J. (2002). The Neuman Systems Model.Fourth Edition. New Jersey: Prentice Hall.

OMS. (2009). Las mujeres y la salud. Los datos de hoy, la agenda de mañana. Ginebra: OMS.

OPS. (2008). Estrategias para el desarrollo de los equipos de APS. Washington D.C.: OPS.

OPS. (2007). La renovación de la atención primaria de salud en las Américas. Washington D.C.: OPS.

Stuart, G. y Laraia, M. (2006). Enfermería psiquiátrica. Principios y práctica. Octava edición. Madrid: Elsevier Mosby.

Ulin, P., Robinson, E. y Tolley, E. (2006). Investigación aplicada en salud pública. Métodos cualitativos. Washington D.C.: OPS.

UNESCO (2005). Declaración Universal sobre Bioética y Derechos Humanos. París: UNESCO.

Vázquez, N. y Murguialday, C. (2000). Con la sartén sin el mango: el trabajo doméstico, base invisible de la economía. San Salvador: Asociación Equipo Maíz.

Wilkinson, R. y Marmot, M. (2003). Determinantes sociales de la salud: los hechos irrefutables. Ginebra: OMS. 